\title{
INDICADORES TEMPRANOS (0-36 MESES) DE RIESGO DE TRASTORNO DEL ESPECTRO AUTISTA. UN ESTUDIO DE REVISIÓN
}

\author{
Raúl Tárraga-Mínguez \\ Facultad de Magisterio. Universidad de Valencia \\ raul.tarraga@uv.es \\ Irene Lacruz-Pérez \\ Universidad de Valencia \\ Gemma Pastor-Cerezuela \\ Universidad de Valencia \\ Må Inmaculada Fernández-Andrés \\ Universidad de Valencia
}

Fecha de Recepción: 13 Junio 2018

Fecha de Admisión: 1 Octubre 2018

\section{RESUMEN}

La prevalencia del trastorno del espectro autista (TEA) ha aumentado de manera muy notable en los últimos años debido fundamentalmente a un aumento del conocimiento sobre el mismo por parte de los profesionales responsables de su identificación y/o diagnóstico.

El presente trabajo tiene como objetivo realizar una revisión de los artículos publicados en 2018 que se han propuesto identificar entre los 0 y 36 meses de vida predictores fiables de la posterior aparición del TEA.

Se realizó una búsqueda en Psyclnfo en la que se localizaron 8 estudios empíricos diseñados para evaluar el poder predictivo de estos indicadores tempranos.

En estos estudios se identificaron diferentes síntomas que se consideran predictores fiables del posterior diagnóstico de TEA, como la existencia de diferencias en el perfil sensorial en comparación con los niños sin TEA, la percepción visual de estímulos de carácter social y diferencias en la capacidad para dirigir la atención y en la adquisición de habilidades motrices finas.

Palabras clave: autismo; indicadores de riesgo; intervención temprana

\section{ABSTRACT \\ EARLY RISK INDICATORS (0-36 MONTHS) OF AUTISM SPECTRUM DISORDER. A REVIEW OF THE LITERATURE \\ The prevalence of the autism spectrum disorder (ASD) has increased significantly in the recent years mainly due to an increase in the knowledge of the professionals responsible of its identifica- tion and/or diagnosis.}




\section{INDICADORES TEMPRANOS (0-36 MESES) DE RIESGO DE TRASTORNO DEL ESPECTRO AUTISTA. UN ESTUDIO DE REVISIÓN}

The objective of the current study is to review the articles published in 2018 that have attempted to identify reliable predictors (between 0 and 36 months of life) of the subsequent onset of ASD.

A search was conducted in Psyclnfo in which 8 empirical studies designed to assess the predictive power of these early indicators were found.

In these studies were identified different symptoms that are considered reliable predictors of the subsequent diagnosis of ASD, such as the existence of differences in the sensory profile compared to children without ASD, the visual perception of social stimuli and differences in the ability of directing the attention and the acquisition of fine motor skills.

Keywords: autism; early intervention; risk indicators

Financiado por el Ministerio de Economía y Competitividad (EDU-2016-78867R, AEI/FEDER, UE) y por la Generalitat Valenciana y el Fondo Social Europeo (GV/2017/084 - ACIF/2016/400)

\section{INTRODUCCIÓN}

El trastorno del espectro autista (TEA) es un trastorno del neurodesarrollo caracterizado por difi-cultades en la comunicación e interacción social en diversos contextos, así como por la presencia de patrones restrictivos y repetitivos de comportamiento, intereses 0 actividades (American Psychiatric Association, 2013). La prevalencia del TEA en los países occidentales ha aumentado de manera muy significativa en los últimos años debido a diferentes factores como la existencia de cambios en los propios criterios diagnósticos, el mayor conocimiento de la temática por parte los profesionales que deben emitir el diagnóstico y la puesta a disposición de los profesionales de un mayor número de instrumentos de evaluación específicos para el TEA (Hansen, Schendel y Parner, 2015).

Estas mejoras en el diagnóstico del TEA son fruto de la realización de grandes esfuerzos en la investigación, unos esfuerzos que también se han orientado en los últimos años al desarrollo de intervenciones educativas, que están evidenciando resultados positivos o prometedores en la reducción de la gravedad de la sintomatología central del TEA o de las dificultades que habitualmente se asocian con el diagnóstico. A este respecto, existen numerosos estudios de revisión sobre diferentes intervenciones que están produciendo resultados prometedores avalados por un número significativo de estudios empíricos. Por ejemplo, Gerow et al. (2018) ofrecen datos sobre la efectividad de las intervenciones basadas en el entrenamiento en comunicación funcional implementado por padres; Makrygianni, Gena, Katoudi y Galanis (2018) han llevado a cabo una revisión en la que se reportan resultados positivos de las intervenciones basadas en análisis conductual aplicado; McGill, Baker y Busse (2014) aportan datos de la eficacia de las intervenciones basadas en historias sociales para reducir conductas desafiantes; Murza, Schwartz, HahsVaughn y Nye (2016) han llevado a cabo una revisión sobre intervenciones orientadas a la mejora de la atención compartida; Parsons, Cordier, Munro, Joosten y Speyer (2017) han conducido un estudio de revisión sobre intervenciones orientadas a la mejora del uso pragmático del lenguaje; y Sanz, Fernández, Pastor y Tárraga (2018) han llevado a cabo una revisión de intervenciones basadas en la metodología TEACCH.

Numerosos estudios remarcan los beneficios de comenzar con estos procesos de intervención lo más tempranamente posible, ya que esto maximiza los beneficios de las intervenciones, especialmente cuando la intervención incluye la formación a padres (Zwaigenbaum et al., 2015). Por ello, uno de los aspectos que se consideran actualmente como prioritarios en la investigación sobre este ámbito es la identificación de síntomas tempranos que puedan ser considerados buenos predicto-res de un posterior diagnóstico de TEA.

Aunque el diagnóstico de TEA no suele realizarse hasta al menos los 3 años, y siempre deben considerarse los diferentes ritmos evolutivos que cada sujeto puede tener durante la infancia, la identificación de estos síntomas tempranos, puede suponer un avance significativo para la implementación de intervenciones educativas tempranas tanto con los propios niños como de formación a sus padres o cuidadores. Estas intervenciones tempranas pueden ser de gran utilidad para redu- 
cir la gravedad de la sintomatología TEA y pueden suponer repercutir en mejoras en el bienestar de los niños y sus familias.

La identificación de estos síntomas tempranos del TEA es compleja por diferentes motivos, entre los que se encuentran: la dificultad de contar con muestras con un elevado número de participantes que puedan aportar robustez a los resultados, la necesidad de aplicar tareas de screening en participantes de muy corta edad, la dificultad que comportan los estudios de seguimiento longitudinal, en los que no es sencillo contar con los mismos participantes en varios momentos y fundamentalmente por la dificultad de tener que identificar a participantes que, por alguna condición, se consideren en riesgo de presentar TEA.

Para solucionar estas dificultades, se han empleado diferentes procedimientos, entre los que destacan los estudios prospectivos con niños menores de 3 años que presenten riesgo de TEA por algún factor de riesgo concreto, siendo el más habitual el tener hermanos mayores con diagnóstico de TEA, ya que cuando un niño es diagnosticado de TEA, el riesgo de que sus hermanos también lo sean aumenta notablemente (Sandin et al., 2014).

Pese a las dificultades intrínsecas que tienen este tipo de estudios, en los últimos años se han venido desarrollando investigaciones en diferentes países, en las que han logrado identificar de manera consistente algunos síntomas tempranos que se asocian significativamente con un aumento a edades posteriores de la sintomatología autista.

El objetivo de este trabajo es revisar y exponer las principales conclusiones de estos estudios longitudinales recientes dedicados a identificar síntomas de detección temprana del TEA o de riesgo posterior de presentar TEA entre los 0 y 3 años de vida.

\section{MÉTODO}

En octubre de 2018 se llevó a cabo una búsqueda en la base de datos PsycINFO usando la siguiente combinación de descriptores: "autism y early"; "autism y screening" y "autism y predict*" en el resumen.

Se limitó la búsqueda de acuerdo a los siguientes criterios: a) artículos científicos revisados por pares, b) publicados en 2018 y c) que incluyeran entre sus participantes a niños de entre 0 y 5 años de edad.

Una vez realizadas las búsquedas, se seleccionaron para la presente revisión únicamente los trabajos que se orientaran a identificar síntomas tempranos no relacionados con factores genéticos, condiciones generales de salud o derivados de la presencia de algún otro síndrome o diagnóstico comórbido. Los resultados de la búsqueda se resumen en la figura 1.

Figura 1.diagrana del proceso de búsqueda de artículos.

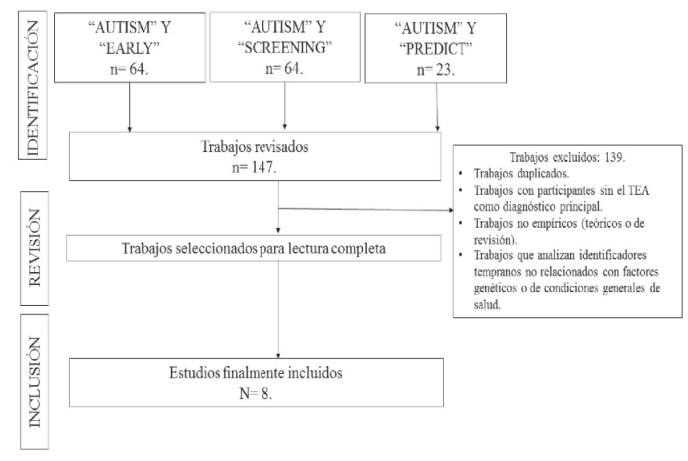




\section{RESULTADOS}

El resumen de la información relevante para la presente revisión de los 8 artículos seleccionados se recoge en la tabla 1.

Se presentan en la tabla 1 los resultados de la revisión de los trabajos Iongitudinales. Estos estudios han sido publicados recientemente en el año 2018.

Los objetivos principales de los artículos revisados estudian la atención, el perfil sensorial, la psicomotricidad, estímulos sociales y no sociales, cribado del trastorno y estímulos biológicos.

Los artículos se presentan según orden alfabético de los primeros autores de los estudios.

En la columna de descripción de los participantes únicamente se incluye información de los grupos de participantes con TEA o con alto riesgo de presentar TEA. Por motivos de aprovechamiento del espacio, no se ofrece la información de los grupos sin diagnóstico o de los grupos de comparación con otros diagnósticos diferentes al TEA.

Tabla 1. Resumen de los estudios incluidos en la revisión.

\begin{tabular}{|c|c|c|c|}
\hline Autor/es (año) & Participantes/pais & Instrumentos/procedimiento & Resultados \\
\hline $\begin{array}{l}\text { Baranek } \\
(2018)\end{array}$ & $\begin{array}{l}87 \text { niños con alto riesgo de } \\
\text { TEA (de acuerdo a los } \\
\text { resultados del First Year } \\
\text { Inventory, FYI). El } \\
\text { diagnóstico se confirmó a } \\
\text { los } 3 \text { años en } 77 \text { niños. } \\
\text { EEUU. }\end{array}$ & $\begin{array}{l}\text { Se evaluó la capacidad para cesar la atención } \\
\text { de un estimulo central para focalizarla en otro } \\
\text { estímulo periférico mediante varios ítems del } \\
\text { FYI y del Autism Observation Scale for } \\
\text { Infants. } \\
\text { La evaluación se realizó a los } 13-15 \text { meses, } \\
20-24 \text { meses y 3-5 años. }\end{array}$ & $\begin{array}{l}\text { Las dificultades para cesar la atención de un estímulo a los 12- } \\
15 \text { meses pueden preceder y predecir un aumento en la } \\
\text { búsqueda de estímulos sensoriales a los } 20-24 \text { meses. Los } \\
\text { resultados apoyan la idea de que los aspectos sensoriales } \\
\text { producen un efecto en cascada en el desarrollo social de los } \\
\text { niños con TEA o con alto riesgo de TEA. }\end{array}$ \\
\hline $\begin{array}{l}\text { Cheung et al. } \\
(2018)\end{array}$ & $\begin{array}{l}113 \text { niños con alto riesgo de } \\
\text { TEA (al menos un hermano } \\
\text { mayor con TEA). } \\
\text { Inglaterra. }\end{array}$ & $\begin{array}{l}\text { Se evaluó la capacidad para retirar la atención } \\
\text { de un estimulo central y fijarla en un estímulo } \\
\text { periférico mediante tareas de ordenador. } \\
\text { Se evaluó a los } 9,15 \text { y } 24 \text { meses de edad de } \\
\text { los participantes. }\end{array}$ & $\begin{array}{l}\text { Los participantes con alto riesgo de TEA cuyo diagnóstico se } \\
\text { confirmó posteriormente obtuvieron un mejor rendimiento en } \\
\text { tareas de búsqueda visual que los participantes sin riesgo de } \\
\text { TEA o con riesgo posteriormente no confirmado. Este mejor } \\
\text { rendimiento predijo la sintomatología TEA a los } 3 \text { años (no } \\
\text { predijo sintomatología de TDAH ni de ansiedad). }\end{array}$ \\
\hline $\begin{array}{l}\text { Choi, Leech, } \\
\text { Tager-Flusberg y } \\
\text { Nelson (2018) }\end{array}$ & 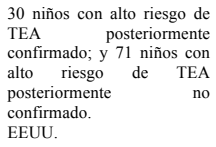 & $\begin{array}{l}\text { Las habilidades motrices se evaluaron a los } 6 \text {, } \\
12 \text {, } 18 \text { y } 24 \text { meses de edad mediante las } \\
\text { Mullen Scales of Early Learning. } \\
\text { La capacidad lingüística se evaluó a los } 36 \\
\text { meses de edad. }\end{array}$ & $\begin{array}{l}\text { Los participantes con alto riesgo de TEA cuyo diagnóstico se } \\
\text { confirmó posteriormente mostraron una mejora } \\
\text { significativamente más lenta en las habilidades de motricidad } \\
\text { fina entre los } 6 \text { y } 24 \text { meses de edad, en comparación a los } \\
\text { participantes sin riesgo o con riesgo de TEA posteriormente } \\
\text { no confirmado. Estas habilidades a los } 6 \text { meses, predecían los } \\
\text { resultados de lenguaje expresivo a los } 3 \text { años de edad. }\end{array}$ \\
\hline $\begin{array}{l}\text { Damiano et al. } \\
(2018)\end{array}$ & $\begin{array}{l}20 \text { niños con alto riesgo de } \\
\text { TEA (al menos un hermano } \\
\text { mayor con TEA). } \\
\text { EEUU }\end{array}$ & $\begin{array}{l}\text { Se evaluó la búsqueda sensorial mediante el } \\
\text { Sensory Processing Assessment a los } 18 \\
\text { meses. } \\
\text { Se evaluó la sintomatología social mediante el } \\
\text { módulo } 2 \text { del ADOS a los } 36 \text { meses. }\end{array}$ & $\begin{array}{l}\text { Los niños con alto riesgo de TEA mostraron más conductas de } \\
\text { búsqueda sensorial que los niños con bajo riesgo de TEA. El } \\
\text { aumento de la búsqueda sensorial se asoció con una reducción } \\
\text { del interés social, y predijo mayores problemas en } \\
\text { sintomatología social. }\end{array}$ \\
\hline $\begin{array}{l}\text { Davidovitch, } \\
\text { Stein, Koren y } \\
\text { Chen (2018) }\end{array}$ & $\begin{array}{l}335 \text { niños con TEA } \\
\text { Israel. }\end{array}$ & $\begin{array}{l}\text { Se realizaron diferentes valoraciones de } \\
\text { cribado de los hitos evolutivos básicos a las: } 6 \\
\text { semanas y } 3,6,9,12,18,24 \text { y } 36 \text { meses de } \\
\text { vida. }\end{array}$ & $\begin{array}{l}\text { Los resultados mostraron que durante los primeros } 6 \text { meses de } \\
\text { vida, los niños con TEA adquirieron los hitos evolutivos } \\
\text { evaluados de acuerdo a la norma. } \\
\text { A los } 9 \text { meses, los niños con TEA ya presentaban dificultades } \\
\text { en el desarrollo del lenguaje, la comunicación y la motricidad, } \\
\text { en comparación a niños con otros diagnósticos y a niños sin } \\
\text { diagnóstico. }\end{array}$ \\
\hline $\begin{array}{l}\text { Falck- Ytter et al. } \\
\text { (2018) }\end{array}$ & $\begin{array}{l}37 \text { niños con alto riesgo de } \\
\text { TEA (al menos un hermano } \\
\text { mayor con TEA); } 20 \text { de ellos } \\
\text { con diagn posteriormente } \\
\text { confirmado. } \\
\text { Suecia. }\end{array}$ & $\begin{array}{l}\text { Se evaluó la preferencia por estímulos de } \\
\text { carácter biológico con sincronía audiovisual a } \\
\text { los } 10 \text { y } 36 \text { meses de edad. }\end{array}$ & $\begin{array}{l}\text { Los niños con TEA mostraron a los } 10 \text { meses una menor } \\
\text { preferencia por estimulos con base biológica } \\
\text { audiovisualmente sincronizados en comparación a los niños } \\
\text { con alto riesgo de TEA posteriormente no confirmado y los } \\
\text { niños con bajo riesgo de TEA. }\end{array}$ \\
\hline $\begin{array}{l}\text { Hendry et al. } \\
(2018) \text {. }\end{array}$ & $\begin{array}{l}113 \text { niños con alto riesgo de } \\
\text { TEA (al menos un hermano } \\
\text { mayor con TEA). } \\
\text { Inglaterra. } \\
\text { Mismos participantes que el } \\
\text { estudio de Cheung et al. } \\
\text { (2018) }\end{array}$ & $\begin{array}{l}\text { Se aplicaron tareas de laboratorio de registro } \\
\text { ocular ante estímulos sociales y no sociales. A } \\
\text { los } 3 \text { años se añadieron } 12 \text { ítems } \\
\text { cumplimentados por los padres para evaluar el } \\
\text { control consciente de la atención. } \\
\text { Se evaluó a los } 9 \text { meses, } 15 \text { meses y } 3 \text { años de } \\
\text { edad de los participantes. }\end{array}$ & $\begin{array}{l}\text { Los participantes con alto riesgo de TEA fijaron la mirada en } \\
\text { estímulos sociales (presentación de rostros humanos), } \\
\text { significativamente menos que los participantes con bajo riesgo } \\
\text { entres los } 9 \text { y } 15 \text { meses de edad. }\end{array}$ \\
\hline $\begin{array}{l}\text { Jones, Dawson y } \\
\text { Webb (2018) }\end{array}$ & $\begin{array}{l}\text { Estudio 1: } 18 \text { niños con } \\
\text { TEA. } \\
\text { Estudio 2: } 43 \text { niños con alto } \\
\text { riesgo de TEA (al menos un } \\
\text { hermano con TEA). } \\
\text { EEUU. }\end{array}$ & $\begin{array}{l}\text { Estudio 1: se evaluó la sensibilidad sensorial } \\
\text { mediante el Short Sensory Profile; y el interés } \\
\text { e interacción social mediante el Pervasive } \\
\text { Developmental Disorder Behavior Inventory } \\
\text { (PDDBI). Se evaluó a los } 2 \text { y } 4 \text { años de edad. } \\
\text { Estudio 2: se evaluó la sensibilidad perceptiva } \\
\text { mediante el Infant Behavior Questionnaire- } \\
\text { Revised. Se evaluó a los } 6,12 \text { y } 18 \text { meses. }\end{array}$ & $\begin{array}{l}\text { Estudio 1: El informe paterno de mayor hipersensibilidad } \\
\text { sensorial a los } 2 \text { años de edad e los niños con TEA predijo el } \\
\text { aumento de la respuesta a estímulos sensoriales y el informe } \\
\text { paterno de interés social a los } 4 \text { años. } \\
\text { Estudio 2: el informe paterno de aumento de sensibilidad } \\
\text { perceptiva a los } 6 \text { meses predijo una mayor atención al } \\
\text { reconocimiento de caras a los } 18 \text { meses. }\end{array}$ \\
\hline
\end{tabular}




\section{DISCUSIÓN}

El análisis de los resultados expuestos en la sección anterior pone de relieve la existencia de diferentes síntomas de aparición temprana que se han erigido como predictores robustos de un diagnóstico posterior de TEA. Entre estos síntomas, destacan los indicadores relacionados con diferencias en aspectos puntuales de perfil sensorial en comparación con los niños sin TEA (Damiano et al., 2018; Jones et al., 2018), la percepción visual de estímulos de carácter social (Falck-Ytter et al. 2018; Hendry et al., 2018), determinados aspectos del funcionamiento ejecutivo relacionados con la capacidad para dirigir la atención (Baranek et al., 2018; Cheung et al., 2018), la adquisición de habilidades motrices finas (Choi et al., 2018), u otros hitos evolutivos (Davidovitch et al., 2018).

La corroboración por parte de futuros estudios de estos mismos indicadores como predictores fiables del TEA, puede contribuir a desarrollar protocolos que permitan identificar tempranamente a niños en riesgo de presentar TEA, y así poder realizar intervenciones psicoeducativas de carácter preventivo desde los primeros meses de vida.

Dado lo complejo de utilizar algunos de los instrumentos empleados en las investigaciones incluidas en la presente revisión, algunos de los cuales precisan de ordenadores y dispositivos para el registro del movimiento ocular, el paso siguiente que debería adoptar esta línea de investigación debería ser el desarrollo de cuestionarios de cribado en los que se solicite a los padres o cuidadores de los niños información relacionada con estos síntomas tempranos, o bien la elaboración de tareas sencillas de cribado que puedan aplicarse en poco tiempo y con bajo coste en protocolos de detección universal. A este respecto, es interesante el First Year Inventory (Reznick et al., 2007), empleado en el estudio de Baranek et al. (2018) incluido en esta revisión, o el protocolo de evaluación de hitos evolutivos seguido en el estudio de Davidovitch et al. (2018).

El presente estudio contribuye a sintetizar en un único documento, algunos de los avances más recientes en la identificación temprana de predictores de un diagnóstico posterior de TEA. Sin embargo, no se trata de un estudio sistemático de todos los posibles indicadores tempranos, ya que se ha revisado únicamente las publicaciones del año 2018, y se han excluido de la revisión los estudios que contemplan factores de riesgo relacionados con aspectos como las complicaciones durante el embarazo 0 el parto, la edad de los padres, o la presencia de otras complicaciones de salud asociadas al TEA. A este respecto, la revisión realizada por Rossignol y Frye, (2012) en la que sintetizan un amplio número de estudios que relacionan el TEA con diferentes condiciones médicas, complementaría las conclusiones del presente estudio.

Finalmente, tras la realización de esta revisión, se abren algunas futuras vías de investigación que sería interesante explorar en el futuro, entre las que se encuentran: el diseño de intervenciones tempranas que puedan ser implementadas en niños con riesgo de presentar TEA en el futuro, y el seguimiento longitudinal de los niños que han recibido estas intervenciones tempranas, para valorar su éxito en la reducción de la gravedad de la sintomatología autista, y para mejorar el bienestar tanto de los niños como de sus familias.

\section{REFERENCIAS BIBLIOGRÁFICAS}

American Psychiatric Association (2013). Diagnostic and statistical manual of mental disorders. 5th ed. Washington, DC: American Psychiatric Association.

*Baranek, G. T., Woynaroski, T. G., Nowell, S., Turner-Brown, L., DuBay, M., Crais, E. R., \& Watson, L. R. (2018). Cascading effects of attention disengagement and sensory seeking on social symptoms in a community sample of infants at-risk for a future diagnosis of autism spectrum disorder. Developmental Cognitive Neuroscience, 29, 30-40. doi: 10.1016/j.dcn.2017.08.006

Chen, M., Su, T., Chen, Y., Hsu, J., Huang, K., Chang, W., . . Bai, Y. (2014). Is atopy in early child- 
hood a risk factor for ADHD and ASD? A longitudinal study. Journal of Psychosomatic Research, 77(4), 316-321. doi: 10.1016/j.jpsychores.2014.06.006

${ }^{*}$ Cheung, C. H. M., Bedford, R., Johnson, M. H., Charman, T., \& Gliga, T. (2018). Visual search performance in infants associates with later ASD diagnosis. Developmental Cognitive Neuroscience, 29, 4-10. doi: 10.1016/j.dcn.2016.09.003

${ }^{*}$ Choi, B., Leech, K. A., Tager-Flusberg, H., \& Nelson, C. A. (2018). Development of fine motor skills is associated with expressive language outcomes in infants at high and low risk for autism spectrum disorder. Journal of Neurodevelopmental Disorders, 10(1), 14. doi: 10.1186/s11689-0189231-3

*Damiano-Goodwin, C. R., Woynaroski, T. G., Simon, D. M., Ibañez, L. V., Murias, M., Kirby, A., ... \& Cascio, C. J. (2018). Developmental sequelae and neurophysiologic substrates of sensory seeking in infant siblings of children with autism spectrum disorder. Developmental Cognitive Neuroscience, 29, 41-53. doi: 10.1016/j.dcn.2017.08.005

*Davidovitch, M., Stein, N., Koren, G., \& Friedman, B. C. (2018). Deviations from Typical Developmental Trajectories Detectable at 9 Months of Age in Low Risk Children Later Diagnosed with Autism Spectrum Disorder. Journal of Autism and Developmental Disorders, 48, 2854-2869. doi: 10.1007/s10803-018-3549-2

*Falck Ytter, T., Nyström, P., Gredebäck, G., Gliga, T., Bölte, S. and EASE Team, (2018). Reduced orienting to audiovisual synchrony in infancy predicts autism diagnosis at 3 years of age. Journal of Child Psychology and Psychiatry, 59(8), 872-880. doi:10.1111/jcpp.12863

Focaroli, V., Taffoni, F., Parsons, S. M., Keller, F., \& Iverson, J. M. (2016). Performance of motor sequences in children at heightened vs. low risk for ASD: A longitudinal study from 18 to 36 months of age. Frontiers in Psychology, 7, 9. Recuperado:https://search.proquest.com/docview/1819919755?accountid $=14777$

Gerow, S., Hagan-Burke, S., Rispoli, M., Gregori, E., Mason, R., y Ninci, J. (2018). A systematic review of parent-implemented functional communication training for children with ASD. Behavior modification, 42(3), 335-363. D0I:10.1177 / 0145445517740872

Hansen, S. N., Schendel, D. E., \& Parner, E. T. (2015). Explaining the increase in the prevalence of autism spectrum disorders: the proportion attributable to changes in reporting practices. JAMA pediatrics, 169(1), 56-62. doi:10.1001/jamapediatrics.2014.1893

*Hendry, A., Jones, E. J., Bedford, R., Gliga, T., Charman, T., \& Johnson, M. H. (2018). Developmental change in look durations predicts later effortful control in toddlers at familial risk for ASD. Journal of Neurodevelopmental Disorders, 10(1), 3. doi: 10.1186/s11689-017-9219-4

*Jones, E. J. H., Dawson, G., \& Webb, S. J. (2018). Sensory hypersensitivity predicts enhanced attention capture by faces in the early development of ASD. Developmental Cognitive Neuroscience, 29, 11-20. doi: 10.1016/j.dcn.2017.04.001

Makrygianni, M. K., Gena, A., Katoudi, S., y Galanis, P. (2018). The effectiveness of applied behavior analytic interventions for children with Autism Spectrum Disorder: A meta-analytic study. Research in Autism Spectrum Disorders, 51, 18-31. https://doi.org/10.1016/j.rasd.2018.03.006

McGill, RJ., Baker, D., \& Busse, R. T. (2015). Social Story interventions for decreasing challenging behaviours: a singlecase meta-analysis 1995-2012. Educational Psychology in Practice, 31(1), 21-42. doi.org/10.1080/02667363.2014.975785

Murza, K. A., Schwartz, J. B., Hahs Vaughn, D. L., y Nye, C. (2016). Joint attention interventions for children with autism spectrum disorder: a systematic review and meta analysis. International Journal of Language \& Communication Disorders, 51(3), 236-251. Doi: 10.1111 / 14606984.12212 
Parsons, L., Cordier, R., Munro, N., Joosten, A., y Speyer, R. (2017). A systematic review of pragmatic language interventions for children with autism spectrum disorder. PloSOne, 12(4), e0172242. doi.org/10.1371/journal.pone.0172242

Sandin, S., Lichtenstein, P., Kuja-Halkola, R., Larsson, H., Hultman, C. M., \& Reichenberg, A. (2014). The familial risk of autism. Jama, 311(17), 1770-1777. doi: 10.1001/jama.2014.4144

Reznick, J. S., Baranek, G. T., Reavis, S., Watson, L. R., \& Crais, E. R. (2007). A parent-report instrument for identifying one-year-olds at risk for an eventual diagnosis of autism: the first year inventory. Journal of Autism and Developmental Disorders, 37(9), 1691-1710. doi: 10.1007/s10803-006-0303-y

Rossignol, D. A., \& Frye, R. E. (2012). A review of research trends in physiological abnormalities in autism spectrum disorders: immune dysregulation, inflammation, oxidative stress, mitochondrial dysfunction and environmental toxicant exposures. Molecular psychiatry, 17(4), 389. doi: doi:10.1038/mp.2011.165

Sanz-Cervera, P., Fernández-Andrés, M. I., Pastor-Cerezuela, G. y Tárraga-Mínguez, R. (2018). Efectividad de las intervenciones basadas en metodología TEACCH en el trastorno del espectro autista: un estudio de revisión. Papeles del Psicólogo, 39(1), 40-50. doi.org/10.23923/pap.psicol2018.2851

Zwaigenbaum, L., Bauman, M. L., Choueiri, R., Kasari, C., Carter, A., Granpeesheh, D., ... \& Pierce, K. (2015). Early intervention for children with autism spectrum disorder under 3 years of age: recommendations for practice and research. Pediatrics, 136(Supplement 1), S60-S81. doi: 10.1542 / peds.2014-3667E 
